\title{
X-ray resonant magnetic scattering from FePd thin films
}

\author{
E. Dudzik, a) S. S. Dhesi, S. P. Collins, H. A. Dürr, and G. van der Laan \\ Magnetic Spectroscopy Group, Daresbury Laboratory, Warrington WA4 4AD, United Kingdom \\ K. Chesnel, M. Belakhovsky, A. Marty, and Y. Samson \\ DRFMC SP2M, CEA-Grenoble, 17 rue des Martyrs, 38054 Grenoble, France
}

\section{J. B. Goedkoop}

University of Amsterdam, Valckenierstraat 65, NL 1018 XE Amsterdam, The Netherlands

\begin{abstract}
Depending on the growth conditions, FePd thin films can display a perpendicular magnetic anisotropy associated with chemical order. In competition with the shape anisotropy, this can lead to striped magnetic domains, with moments perpendicular to the film plane. Under these circumstances, magnetic flux closure should occur. The striped domains were studied with soft x-ray resonant magnetic scattering using circularly polarized light to demonstrate the presence of closure domains. Magnetic depth profiling was performed both at the Fe and $\mathrm{Pd} L_{3}$ edge, by measuring the magnetic diffraction peak intensities versus angle of incidence $\theta$. (C) 2000 American Institute of Physics. [S0021-8979(00)65408-7]
\end{abstract}

Resonant x-ray magnetic scattering makes use of the strong cross-section enhancement of the (otherwise very weak) magnetic scattering that occurs when the incident $\mathrm{x}$-ray energy is tuned across a core-level absorption edge. ${ }^{1}$ This technique can be used to study periodic magnetic structures, provided that their dimensions are in the order of the $\mathrm{x}$-ray wavelength. We report on various ways for obtaining magnetic depth profiles from thin film samples using soft $\mathrm{x}$-ray magnetic scattering (SOXMAS).

In the work described here FePd thin films with perpendicular magnetic anisotropy (PMA) were studied using SOXMAS at both the Fe and $\mathrm{Pd} L_{3}$ edges at 709 and $3174 \mathrm{eV}$, respectively. The films were grown by co-depositing $\mathrm{Fe}$ and Pd onto a Pd buffer layer on an $\mathrm{MgO}$ substrate. Depending on the growth temperature, the resulting films are more or less chemically ordered, ${ }^{2}$ with a PMA proportional to the degree of chemical order. In samples with moderate anisotropy $\left(Q=K_{u} / 2 \pi M_{s}^{2} \sim 0.8\right.$, where $K_{u}$ is the perpendicular anisotropy constant and $M_{s}$ the saturation magnetization per volume unit $)^{3}$ the competition between PMA and shape anisotropy leads to the spontaneous formation of striped domains with a magnetization profile $\uparrow \downarrow \uparrow \downarrow \uparrow$ perpendicular to the film plane. This magnetic structure is characterized by magnetic flux lines partially outside the sample. Under these conditions, internal flux line closure is expected to occur with closure domains at the sample surface. ${ }^{4}$ The resulting magnetization profile should therefore be chiral, i.e., $\uparrow \rightarrow \downarrow$ $\leftarrow \uparrow \rightarrow \downarrow \leftarrow$. We will describe a method of detecting such chiral structures which makes use of the magnetic x-ray circular dichroism effect (i.e., the difference in the scattering amplitude between left and right circularly polarized light) in the diffraction pattern.

The experiments were conducted at the European Synchrotron Radiation Facility in Grenoble on stations ID12B and ID12A, using both linearly and circularly polarized light.

\footnotetext{
a) Author to whom correspondence should be addressed, electronic mail: e.dudzik@dl.ac.uk
}

A Daresbury two-circle diffractometer chamber was used throughout. Experiments were done in two geometries: in geometry A, the striped domains were aligned perpendicularly to the scattering plane, and the detector was mounted on the exit arm of the diffractometer. In this geometry, "conventional" $\theta-2 \theta$ reflectivity curves could be obtained, as well as rocking scans where the detector was kept at a fixed angle while the angle of incidence $\theta$ on the sample was varied. In geometry B, the striped domains were parallel to the scattering planes, and the detector was mounted on a motorized arm which could be scanned perpendicularly across the scattering plane. This arm also allowed a restricted range of movement for the $2 \theta$ exit angle, so that a series of transverse scans could be measured for a range of angles of incidence, $\theta$.

Figure 1 shows diffraction scans taken at the $\mathrm{Fe} L_{3}$ edge in geometries A and B. In both cases the scans show satellites around the central specular peak. These satellite peaks occur at multiples of $0.007 \AA^{-1}$ in the momentum transfer, which corresponds to a real space periodicity of $910 \AA$, in very good agreement with the striped domain period determined by magnetic force microscopy (MFM). These satellite peaks can therefore be ascribed purely to the magnetic structure, so that they can be used to obtain detailed information about the magnetic properties of the sample.

The scans shown in Fig. 1 were measured with both left and right circularly polarized light. In geometry A there is no difference in the scattering for both polarizations, while geometry B clearly shows circular dichroism, with an opposite sign on either side of the specular peak. This can be understood in terms of the selection rules for light polarized parallel ( $\pi$-polarization) or perpendicularly ( $\sigma$-polarization) to the scattering plane, using the fact that the unit vectors of the incoming and outgoing light polarization and the magnetization should result in a nonvanishing product $\left(\hat{e}_{\text {out }} \times \hat{e}_{\text {in }}\right) \cdot \hat{M}$. When the striped domains are parallel to the scattering planes, the closure domain magnetization is perpendicular to the scattering plane, while the PMA domain magnetization 


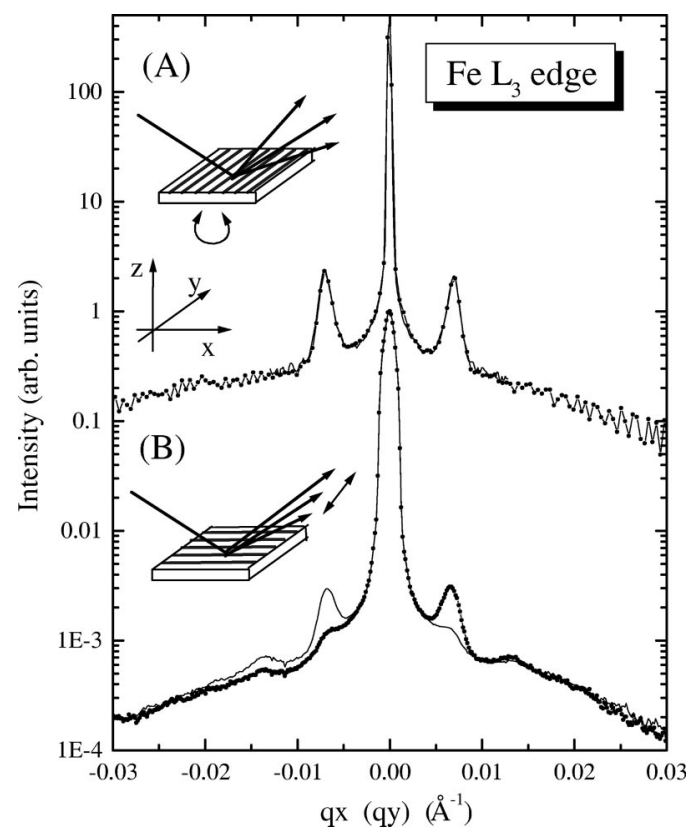

FIG. 1. Magnetic satellite peaks observed around the specular peak in scattering geometries A (top) and B (bottom) at $709 \mathrm{eV}$ photon energy, with left (dotted line) and right (solid line) circularly polarized light. X-ray circular dichroism only occurs in geometry B. The coordinate system shown here is used throughout the article.

lies in the scattering plane. This leads to two contributions to the $\pi$-polarized scattered light, one from the $\pi \rightarrow \pi$ scattering by the closure domains, the other from $\sigma \rightarrow \pi$ scattering by the PMA domains. Since the closure domains are offset relative to the PMA domains, the two $\pi$ components have a relative phase difference of $90^{\circ}$. This phase difference manifests itself when circularly polarized light is used, because the $\pm 90^{\circ}$ phase difference between the $\sigma$ and the $\pi$ component of the circularly polarized light is added to the $90^{\circ}$ phase shift caused by the scattering from the ordered PMA and closure domains, leading to a total phase shift of $\pm 180^{\circ}$, and thus to constructive and destructive interference. In geometry A, on the other hand, both PMA and closure domain magnetization lie in the scattering plane, so that no $\pi \rightarrow \pi$ scattering occurs. The presence of circular dichroism in ge-

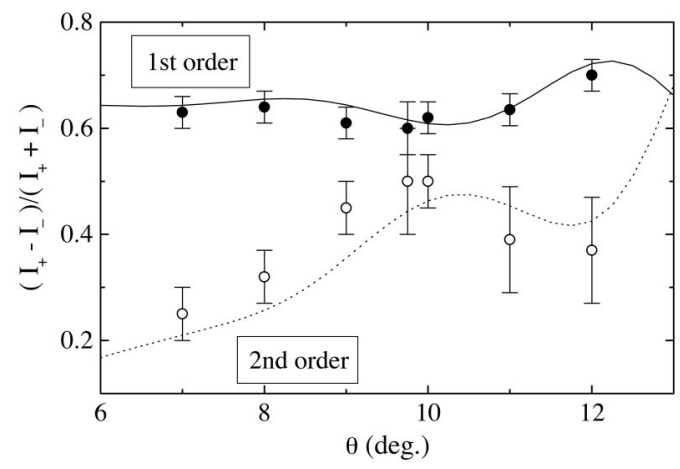

FIG. 2. Asymmetry ratio $\left(I_{+}-I_{-}\right) /\left(I_{+}+I_{-}\right)$of the dichroism signal plotted against angle of incidence, $\theta$. This information can be used to obtain a perpendicular magnetic profile of the sample. Dotted and solid lines are fits to a theoretical model.

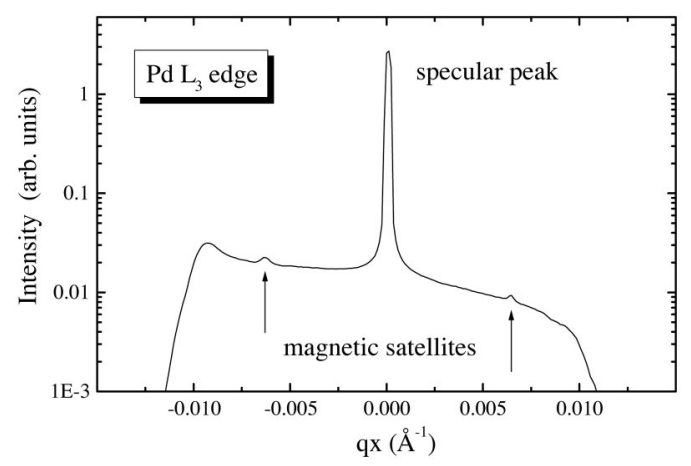

FIG. 3. Magnetic satellite peaks observed around the specular peak in geometry A at the $\operatorname{Pd} L_{3}$ edge.

ometry B clearly evidences the presence of a chiral domain structure.

Figure 2 shows the variation of the asymmetry ratio $\left(I_{+}-I_{-}\right) /\left(I_{+}+I_{-}\right)$of the circular dichroism signal with the angle of incidence $\theta$. This dependence allows us to model the depth profile of the magnetization. This depth profile was approximated by assuming a surface layer with both PMA and closure domains, and a bulk layer containing only PMA domains, ${ }^{5}$ the periodic magnetization was modeled using the first two terms of a Fourier series. The polarization dependent resonant magnetic scattering cross sections were taken from literature. ${ }^{6}$ In this way it was possible to determine the depth of the closure domains. The fits shown in Fig. 2 correspond to an effective depth of $125 \AA$ in a 420 - $\AA$-thick FePd film.

At the Pd $L_{3}$ edge, the size of the magnetic scattering amplitude is much smaller than at the $\mathrm{Fe} L_{3}$ edge, because the Pd magnetic moment $\left(0.65 \mu_{B}\right)$ is less than a third of the Fe moment $\left(2.2 \mu_{B}\right),{ }^{7}$ which reduces the magnetic scattering signal by a factor of 10 relative to the charge scattering. Figure 3 shows a rocking scan taken in geometry A at 3174 $\mathrm{eV}$. The two magnetic satellite peaks are visible, but much weaker in comparison to the specular intensity than at the $\mathrm{Fe}$ edge.

Magnetic depth profiles can also be obtained in geometry A, by measuring the intensity of the magnetic satellite peaks as a function of $\theta$. At the Fe edge such magnetic diffraction rod scans were performed by scanning both sample angle $\theta$ and detector angle $2 \theta$ simultaneously, so that

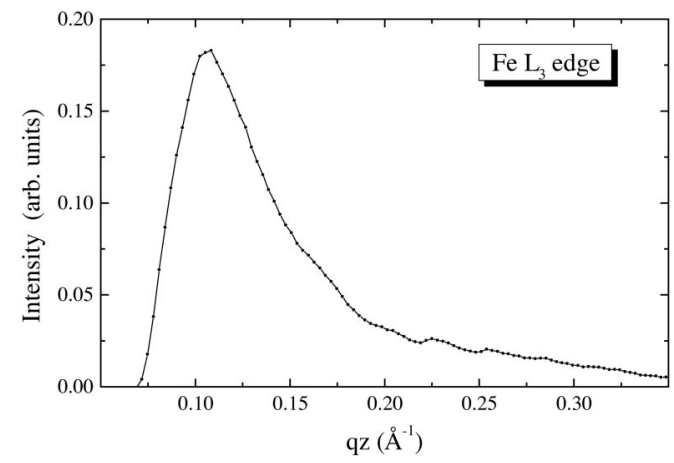

FIG. 4. Rod scan of the magnetic satellite peak taken at the Fe $L_{3}$ edge, obtained with fixed momentum transfer $q_{x}=0.007 \AA^{-1}$. 


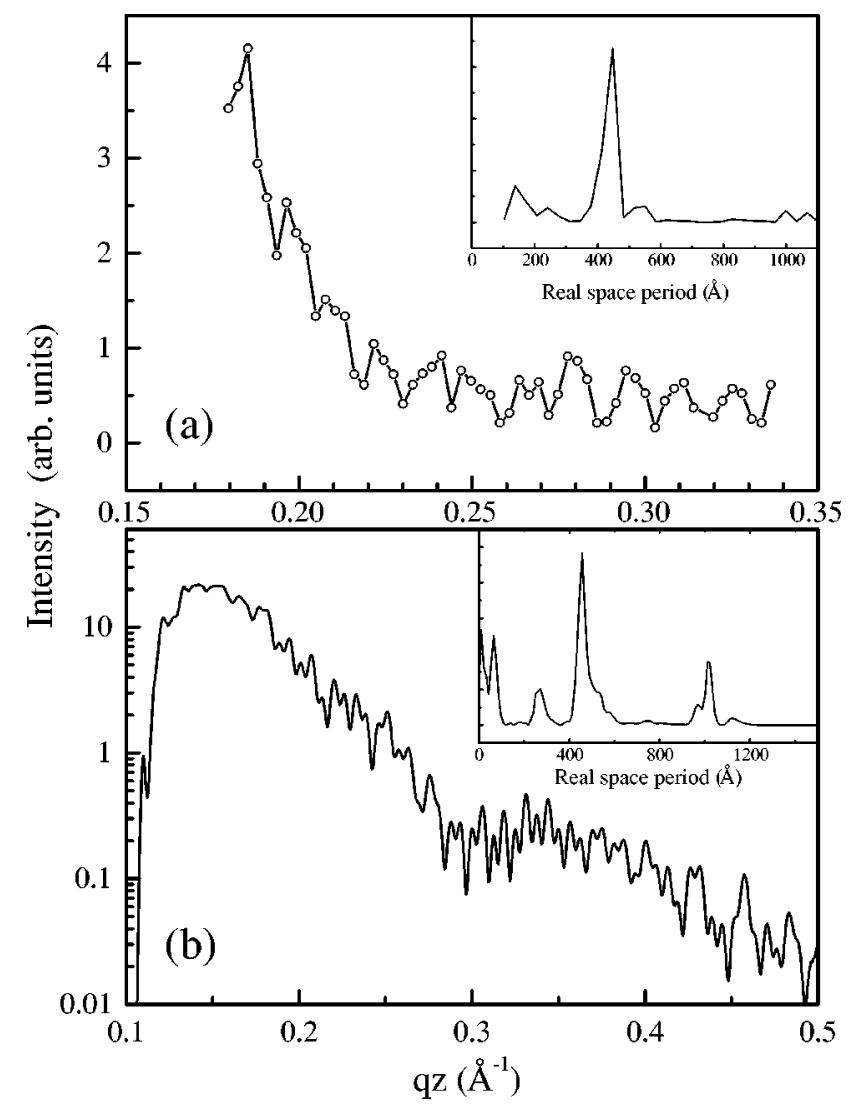

FIG. 5. Rod scans of the magnetic satellite peak at $q_{x}=0.0069 \AA^{-1}$ (a), and of the specular reflectivity (b). The insets show the Fourier transform of the rod data.

the momentum transfer $q_{x}$ was kept constant at $0.007 \AA^{-1}$. The resulting scan is shown in Fig. 4. At the Pd edge, rod scans had to be measured by rocking $\theta$ across the magnetic peak for a series of exit angles $2 \theta$. The diffuse background was then subtracted and the peak intensity integrated.

Figure 5 shows both a magnetic rod scan taken at the Pd $L_{3}$ edge and a (nonmagnetic) specular reflectivity scan. While the Fe magnetic rod scan shows only a broad intensity change with $q_{z}$, periodic intensity modulations can be seen in the two Pd edge scans. The insets in Fig. 5 show the Fourier transforms of the two rod scans. The specular reflectivity has two main Fourier components with real space periods of 1020 and $420 \AA$, while the magnetic rod scan has only a single component at a real space period of $420 \AA$. The $420 \AA$ period corresponds to the thickness of the magnetic FePd layer, while the longer $1020 \AA$ period in the specular data corresponds to the combined thickness of the Pd capping, FePd and Pd buffer layers. While the specular reflectivity shows Kiessig fringes from both the combined and the FePd film thickness, the magnetic rod scan shows purely magnetic Kiessig fringes caused by the interference of the reflections from the top and bottom of the FePd magnetic layer. Although the sampling period of the magnetic rod data is actually too low to allow the detection of high frequency components, the $420 \AA$ Kiessig fringes clearly dominate the scan. The lack of fringes in the Fe edge rod scan (Fig. 4), on the other hand, is linked to the comparatively small penetration depth of the $709 \mathrm{eV} x$ rays, which means that there is virtually no reflectivity from below a few hundred $\AA$ deep. It is possible to model the magnetic rod scans in a similar way as in the model presented by Dürr et al. ${ }^{5}$, leading to a precise description of the magnetic domain structure in the FePd thin films.

${ }^{1}$ D. Gibbs, D. R. Harshmann, E. D. Isaacs, D. B. McWhan, D. Mills, and C. Vettier, Phys. Rev. Lett. 61, 1241 (1988).

${ }^{2}$ V. Gehanno, A. Marty, B. Gilles, and Y. Samson, Phys. Rev. B 55, 12552 (1997).

${ }_{3}^{3}$ A. Marty, Y. Samson, B. Gilles, M. Belakhovsky, E. Dudzik, H. A. Dürr, S. S. Dhesi, G. van der Laan, and J. B. Goedkoop, J. Appl. Phys. (these proceedings, Fig. 1).

${ }^{4}$ C. Kittel, Phys. Rev. 70, 965 (1946).

${ }^{5}$ H. A. Dürr, E. Dudzik, S. S. Dhesi, J. B. Goedkoop, G. van der Laan, M. Belakhovsky, C. Mocuta, A. Marty, and Y. Samson, Science 284, 2166 (1999).

${ }^{6}$ J. P. Hill and D. F. McMorrow, Acta Crystallogr., Sect. A: Found Crystallogr. 52, 236 (1996).

${ }^{7}$ P. Kamp, A. Marty, R. Hoffmann, S. Marchesini, M. Belakhovsky, C. Boeglin, H. A. Dürr, S. S. Dhesi, G. van der Laan, and A. Rogalev, Phys. Rev. B 59, 1105 (1999). 